\title{
Convolution identities for Bernoulli and Genocchi polynomials
}

\author{
Takashi Agoh* \\ Department of Mathematics \\ Tokyo University of Science \\ Noda, Chiba 278-8510, Japan \\ agoh_takashi@ma.noda.tus.ac.jp
}

Submitted: Jun 20, 2013; Accepted: Mar 14, 2014; Published: Mar 24, 2014

Mathematics Subject Classifications: 11B68, 11B83

\begin{abstract}
The main purpose of this paper is to derive various Matiyasevich-Miki-Gessel type convolution identities for Bernoulli and Genocchi polynomials and numbers by applying some Euler type identities with two parameters.
\end{abstract}

Keywords: Bernoulli polynomial; Genocchi polynomial; Euler's identity; Miki's identity; beta integral.

\section{Introduction}

The Bernoulli numbers $B_{n}$ and polynomials $B_{n}(x)$ appear in many areas of mathematics and theoretical physics, most notably in number theory, the calculus of finite differences, asymptotic analysis and quantum field theory. They can be defined by the generating functions

$$
\begin{aligned}
P(t) & :=\frac{t}{e^{t}-1}=\sum_{n=0}^{\infty} B_{n} \frac{t^{n}}{n !} \quad(|t|<2 \pi), \\
P(t, x) & :=\frac{t e^{x t}}{e^{t}-1}=\sum_{n=0}^{\infty} B_{n}(x) \frac{t^{n}}{n !} \quad(|t|<2 \pi),
\end{aligned}
$$

respectively. As is easily shown, we see $B_{n}(0)=(-1)^{n} B_{n}(1)=B_{n}$ and $B_{n}(1-x)=$ $(-1)^{n} B_{n}(x)$ for $n \geqslant 0$.

\footnotetext{
${ }^{*}$ Supported by the Ministry of Education, Science, Sports and Culture, Grant-in-Aid for Scientific Research (C).
} 
On the other hands, the Genocchi numbers $G_{n}$ and polynomials $G_{n}(x)$ are defined by the generating functions

$$
\begin{gathered}
Q(t):=\frac{2 t}{e^{t}+1}=\sum_{n=0}^{\infty} G_{n} \frac{t^{n}}{n !} \quad(|t|<\pi), \\
Q(t, x):=\frac{2 t e^{x t}}{e^{t}+1}=\sum_{n=0}^{\infty} G_{n}(x) \frac{t^{n}}{n !} \quad(|t|<\pi),
\end{gathered}
$$

respectively. It is easily seen that $G_{2 k+1}=0,(-1)^{k} G_{2 k}>0$ for $k \geqslant 1$ and $G_{n}(0)=G_{n}$. They can be expressed in terms of Bernoulli numbers and polynomials by $G_{n}=2\left(1-2^{n}\right) B_{n}$ and $G_{n}(x)=2 B_{n}(x)-2^{n+1} B_{n}(x / 2)$. Further, we can express them in terms of Euler numbers $E_{n}$ and polynomials $E_{n}(x)$ defined by

$$
\begin{aligned}
& R(t):=\frac{2}{e^{t}+e^{-t}}=\sum_{n=0}^{\infty} E_{n} \frac{t^{n}}{n !} \quad(|t|<\pi), \\
& R(t, x):=\frac{2 e^{x t}}{e^{t}+1}=\sum_{n=0}^{\infty} E_{n}(x) \frac{t^{n}}{n !} \quad(|t|<\pi),
\end{aligned}
$$

respectively. Indeed, we see that $G_{n+1}(x)=(n+1) E_{n}(x)(n \geqslant 0)$ and

$$
G_{n+1}=\frac{n+1}{2^{n}} \sum_{i=0}^{n}\left(\begin{array}{l}
n \\
i
\end{array}\right)(-1)^{n-i} E_{i} \quad(n \geqslant 0) .
$$

Concerning convolution identities for Bernoulli numbers, the most basic and remarkable one is the following formula, which is usually attributed to Euler:

$$
\sum_{i=0}^{n}\left(\begin{array}{l}
n \\
i
\end{array}\right) B_{i} B_{n-i}=-n B_{n-1}-(n-1) B_{n} \quad(n \geqslant 1) .
$$

This identity was extended to Bernoulli polynomials and generalized in many directions (see, e.g., [1, 2, 3, 4, 5, 7, 13]). For instance, we have, as a simple one,

$$
\sum_{i=0}^{n}\left(\begin{array}{l}
n \\
i
\end{array}\right) B_{i}(x) B_{n-i}(y)=n(x+y-1) B_{n-1}(x+y)-(n-1) B_{n}(x+y) \quad(n \geqslant 1) .
$$

In 1978, a very different type of convolution identity for Bernoulli numbers was proved by Miki [16] based on $p$-adic arguments:

$$
\sum_{i=2}^{n-2} \frac{B_{i} B_{n-i}}{i(n-i)}-\sum_{i=2}^{n-2}\left(\begin{array}{l}
n \\
i
\end{array}\right) \frac{B_{i} B_{n-i}}{i(n-i)}=2 H_{n} \frac{B_{n}}{n} \quad(n \geqslant 4),
$$

where $H_{n}$ is the $n$th harmonic number defined by

$$
H_{n}:=1+\frac{1}{2}+\cdots+\frac{1}{n}
$$


Subsequently, Matiyasevich [15] discovered a good companion identity to (1.6) with the aid of the computer software system "Mathematica". Rewriting his original identity in an equivalent form,

$$
\sum_{i=2}^{n-2} B_{i} B_{n-i}-2 \sum_{i=2}^{n-2}\left(\begin{array}{c}
n+1 \\
i-1
\end{array}\right) \frac{B_{i} B_{n-i}}{i}=\frac{n(n+1)}{n+2} B_{n} \quad(n \geqslant 4) .
$$

This identity was later proved and generalized by several authors (cf., e.g., [8, 10, 17]).

In 2005, Miki's identity (1.6) was extended to Bernoulli polynomials by Gessel [12] using the Stirling numbers of the second kind. Indeed, he proved that for $n \geqslant 1$,

$$
\sum_{i=1}^{n-1} \frac{B_{i}(x) B_{n-i}(x)}{i(n-i)}-\frac{2}{n} \sum_{i=0}^{n-1}\left(\begin{array}{c}
n \\
i
\end{array}\right) \frac{B_{i}(x) B_{n-i}}{n-i}-B_{n-1}(x)=2 H_{n-1} \frac{B_{n}(x)}{n} .
$$

In this paper, we study the Matiyasevich-Miki-Gessel (we simply write as "M-M-G") type convolution identities for Bernoulli and Genocchi polynomials. In Section 2, we deduce some Euler type identities with two parameters for these polynomials by observing certain functional equations related to the generating functions $P(t, x)$ and $Q(t, x)$. Considering special cases of these Euler type identities, we derive in Section 3 various M-M-G type identities for Bernoulli and Genocchi polynomials and numbers.

The basic idea we will use in Section 3 is the following. Given two functions $F(t):=$ $\sum_{n=0}^{\infty} f_{n} t^{n} / n$ ! and $G(t):=\sum_{n=0}^{\infty} g_{n} t^{n} / n$ !, suppose that we have an identity

$$
F(u t) G((1-u) t)=\sum_{n=0}^{\infty} h_{n}(u) \frac{t^{n}}{n !}, \quad u \in \mathbb{R} .
$$

Equating coefficients of $t^{n} / n$ ! on both sides and integrating with respect to $u$ from 0 to 1 , one can deduce

$$
\frac{1}{n+1} \sum_{k=0}^{n} f_{k} g_{n-k}=\int_{0}^{1} h_{n}(u) d u .
$$

Here we have used the beta integral $\int_{0}^{1} u^{k}(1-u)^{n-k} d u=k !(n-k) ! /(n+1)$ ! (Lemma 2-(i) below). This is essentially the same idea used by Crabb in [6] to give another short and intelligible proof of (1.7).

\section{Euler type convolution identities}

For arbitrary two sequences $S=\left\{S_{n}\right\}_{n \geqslant 0}$ and $T=\left\{T_{n}\right\}_{n \geqslant 0}$, we will use the following umbral notation for simplification. For $u, v \in \mathbb{R}$, letting $0^{0}=1$ by convention (in the case of $u=0$ or $v=0$ ), we define the notation $[u S+v T]^{n}$ by

$$
[u S+v T]^{n}:=\sum_{i=0}^{n}\left(\begin{array}{l}
n \\
i
\end{array}\right) u^{i} v^{n-i} S_{i} T_{n-i} \quad(n \geqslant 0)
$$


We now establish some Euler type convolution identities with two parameters for Bernoulli and Genocchi polynomials by observing certain functional equations satisfied by the generating functions $P(t, x)$ and $Q(t, x)$ of these polynomials.

Theorem 1. Let $u, v \in \mathbb{R} \backslash\{0\}$ with $u+v \neq 0$ and put $W:=(u x+v y) /(u+v)$. For $n \geqslant 1$, we have

$$
\begin{aligned}
{[u B(x)+v B(y)]^{n}=} & u v(u+v)^{n-2} n B_{n-1}(W)+\frac{v}{u+v}[(u+v) B(W)+u B]^{n} \\
& +\frac{u}{u+v}[(u+v) B(W)+v B]^{n}, \\
{[u G(x)+v G(y)]^{n}=} & 4 u v(u+v)^{n-2} n B_{n-1}(W)-\frac{2 v}{u+v}[(u+v) B(W)+u G]^{n} \\
& -\frac{2 u}{u+v}[(u+v) B(W)+v G]^{n}, \\
{[u B(x)+v G(y)]^{n}=} & u v(u+v)^{n-2} n G_{n-1}(W)+\frac{v}{u+v}[(u+v) G(W)+u B]^{n} \\
& -\frac{u}{2(u+v)}[(u+v) G(W)+v G]^{n} .
\end{aligned}
$$

Proof. We will use the following rational function identities of $X$ which can be easily confirmed by direct calculations:

$$
\begin{aligned}
& \frac{1}{X^{u}-1} \cdot \frac{1}{X^{v}-1}=\frac{1}{X^{u+v}-1}\left(1+\frac{1}{X^{u}-1}+\frac{1}{X^{v}-1}\right) \quad(u v(u+v) \neq 0), \\
& \frac{1}{X^{u}+1} \cdot \frac{1}{X^{v}+1}=\frac{1}{X^{u+v}-1}\left(1-\frac{1}{X^{u}+1}-\frac{1}{X^{v}+1}\right) \quad(u+v \neq 0) \text {, } \\
& \frac{1}{X^{u}-1} \cdot \frac{1}{X^{v}+1}=\frac{1}{X^{u+v}+1}\left(1+\frac{1}{X^{u}-1}-\frac{1}{X^{v}+1}\right) \quad(u \neq 0) \text {. }
\end{aligned}
$$

Here we put $X=e^{t}$ and multiply (a), (b) and (c) by $\kappa:=u v t^{2} e^{(u x+v y) t}, 4 \kappa$ and $2 \kappa$, respectively. Then we can obtain the following functional equations:

$$
\begin{aligned}
P(u t, x) P(v t, y)= & \frac{u v}{u+v} t P((u+v) t, W)+\frac{v}{u+v} P((u+v) t, W) P(u t) \\
& \quad+\frac{u}{u+v} P((u+v) t, W) P(v t), \\
Q(u t, x) Q(v t, y)= & \frac{4 u v}{u+v} t P((u+v) t, W)-\frac{2 v}{u+v} P((u+v) t, W) Q(u t) \\
& \quad-\frac{2 u}{u+v} P((u+v) t, W) Q(v t), \\
P(u t, x) Q(v t, y)= & \frac{u v}{u+v} t Q((u+v) t, W)+\frac{v}{u+v} Q((u+v) t, W) P(u t) \\
& -\frac{u}{2(u+v)} Q((u+v) t, W) Q(v t) .
\end{aligned}
$$


Equating coefficients of $t^{n} / n$ ! in the power series expansions on both sides of these equations, we can deduce the Euler type formulas as indicated.

Many kinds of convolution identities for Bernoulli and Genocchi polynomials can be obtained from Theorem 1 by taking various values of $u$ and $v$. In particular, considering the special case when $u+v=1$ and $x=y$, we will derive M-M-G type convolution identities for these polynomials and numbers in the next section.

\section{M-M-G type convolution identities}

We first present the following lemma which will be needed throughout in this section:

Lemma 2. Let $H_{n}$ be the $n$th harmonic number defined in (1.5). It follows that

$$
\begin{aligned}
& \int_{0}^{1} u^{n-1}(1-u)^{k-1} d u=\frac{(n-1) !(k-1) !}{(n+k-1) !} \quad(n, k \geqslant 1), \\
& \frac{1}{2} \int_{0}^{1} \frac{1-u^{n+1}-(1-u)^{n+1}}{u(1-u)} d u=\int_{0}^{1} \frac{1-u^{n}}{1-u} d u=H_{n} \quad(n \geqslant 1) .
\end{aligned}
$$

We do not give the proof of this lemma, but both formulas can be easily verified by elementary calculations. Here note that (i) is the well-known beta integral and (ii) is found in Crabb's paper [6] (where it is used in exactly the same way).

Put $y=x$ and $v=1-u(\neq 0)$ in Theorem 1 . Then, since $W=(x u+v y) /(u+v)=x$, we have the identities

$$
\begin{gathered}
{[u B(x)+(1-u) B(x)]^{n}=u(1-u) n B_{n-1}(x)} \\
+(1-u)[B(x)+u B]^{n}+u[B(x)+(1-u) B]^{n}, \\
{[u G(x)+(1-u) G(x)]^{n}=4 u(1-u) n B_{n-1}(x)} \\
\quad-2(1-u)[B(x)+u G]^{n}-2 u[B(x)+(1-u) G]^{n} \\
{[u B(x)+(1-u) G(x)]^{n}=u(1-u) n G_{n-1}(x)} \\
+(1-u)[G(x)+u B]^{n}-\frac{u}{2}[G(x)+(1-u) G]^{n} .
\end{gathered}
$$

Applying above (3.1), we can deduce the following polynomial versions of Matiyasevich's and Miki's identities mentioned in Section 1.

Theorem 3. For $n \geqslant 1$, we have

$$
\begin{aligned}
\sum_{i=1}^{n-1} B_{i}(x) B_{n-i}(x) & -\frac{2}{n+2} \sum_{i=0}^{n-1}\left(\begin{array}{c}
n+2 \\
i
\end{array}\right) B_{i}(x) B_{n-i} \\
& =\frac{n(n+1)}{6} B_{n-1}(x)+(n-1) B_{n}(x)
\end{aligned}
$$


(ii)

$$
\begin{aligned}
\sum_{i=1}^{n-1} \frac{B_{i}(x) B_{n-i}(x)}{i(n-i)} & -\frac{2}{n} \sum_{i=0}^{n-1}\left(\begin{array}{c}
n \\
i
\end{array}\right) \frac{B_{i}(x) B_{n-i}}{n-i} \\
& =B_{n-1}(x)+2 H_{n-1} \frac{B_{n}(x)}{n} \quad((1.7), \text { Gessel }) .
\end{aligned}
$$

Proof. We rewrite (3.1) gathering the terms involving $B_{n}(x)$ in one place as

$$
\begin{aligned}
\sum_{i=1}^{n-1}\left(\begin{array}{c}
n \\
i
\end{array}\right) & u^{i}(1-u)^{n-i} B_{i}(x) B_{n-i}(x) \\
& \quad-\sum_{i=0}^{n-1}\left(\begin{array}{c}
n \\
i
\end{array}\right)\left(u^{n-i}(1-u)+u(1-u)^{n-i}\right) B_{i}(x) B_{n-i} \\
= & u(1-u) n B_{n-1}(x)+\left(1-u^{n}-(1-u)^{n}\right) B_{n}(x) .
\end{aligned}
$$

Integrating this between 0 and 1 with respect to $u$, we obtain using Lemma 2-(i),

$$
\begin{gathered}
\frac{1}{n+1} \sum_{i=1}^{n-1} B_{i}(x) B_{n-i}(x)-2 \sum_{i=0}^{n-1}\left(\begin{array}{c}
n \\
i
\end{array}\right) \frac{B_{i}(x) B_{n-i}}{(n+2-i)(n+1-i)} \\
=\frac{n}{6} B_{n-1}(x)+\frac{n-1}{n+1} B_{n}(x) .
\end{gathered}
$$

Since $\frac{1}{(n+2-i)(n+1-i)}\left(\begin{array}{c}n \\ i\end{array}\right)=\frac{1}{(n+1)(n+2)}\left(\begin{array}{c}n+2 \\ i\end{array}\right)$, we know that this identity implies (i) multiplying by $n+1$. Next, we divide $(3.4)$ by $u(1-u)$. Then we get

$$
\begin{gathered}
\sum_{i=1}^{n-1}\left(\begin{array}{c}
n \\
i
\end{array}\right) u^{i-1}(1-u)^{n-1-i} B_{i}(x) B_{n-i}(x)-\sum_{i=0}^{n-1}\left(\begin{array}{c}
n \\
i
\end{array}\right)\left(u^{n-1-i}+(1-u)^{n-1-i}\right) B_{i}(x) B_{n-i} \\
=n B_{n-1}(x)+\frac{1-u^{n}-(1-u)^{n}}{u(1-u)} B_{n}(x) .
\end{gathered}
$$

In the same way as above, integrating this identity between 0 and 1 with respect to $u$, we obtain using both formulas in Lemma 2,

$$
n \sum_{i=1}^{n-1} \frac{B_{i}(x) B_{n-i}(x)}{i(n-i)}-2 \sum_{i=0}^{n-1}\left(\begin{array}{c}
n \\
i
\end{array}\right) \frac{B_{i}(x) B_{n-i}}{n-i}=n B_{n-1}(x)+2 H_{n-1} B_{n}(x),
$$

which gives (ii) dividing by $n$. This completes the proof.

As an immediate consequence of Theorem 3, we can state the following

Corollary 4. For $n \geqslant 4$, we have

$$
\begin{aligned}
& \sum_{i=2}^{n-2} B_{i} B_{n-i}-2 \sum_{i=2}^{n-2}\left(\begin{array}{c}
n+1 \\
i-1
\end{array}\right) \frac{B_{i} B_{n-i}}{i}=\frac{n(n+1)}{n+2} B_{n} \quad((1.6), \text { Matiyasevich }), \\
& \sum_{i=2}^{n-2} \frac{B_{i} B_{n-i}}{i(n-i)}-\sum_{i=2}^{n-2}\left(\begin{array}{c}
n \\
i
\end{array}\right) \frac{B_{i} B_{n-i}}{i(n-i)}=2 H_{n} \frac{B_{n}}{n} \quad((1.4), \text { Miki }) .
\end{aligned}
$$


Proof. If $n \geqslant 4$ is odd, then both sides of (i) and (ii) entirely vanish. So it suffices to consider the case when $n \geqslant 4$ is even. Taking $x=0$ in Theorem 3 -(i), we have, since $B_{0}=1$ and $B_{n-1}=0$,

$$
\sum_{i=2}^{n-2} B_{i} B_{n-i}-\frac{2}{n+2}\left(\sum_{i=2}^{n-2}\left(\begin{array}{c}
n+2 \\
i
\end{array}\right) B_{i} B_{n-i}+B_{n}\right)=(n-1) B_{n},
$$

which gives (i) because $\left(\begin{array}{c}n+2 \\ i\end{array}\right)=\frac{n+2}{i}\left(\begin{array}{c}n+1 \\ i-1\end{array}\right)$. Similarly, taking $x=0$ in Theorem 3-(ii), we have

$$
\sum_{i=2}^{n-2} \frac{B_{i} B_{n-i}}{i(n-i)}-\frac{2}{n}\left(\sum_{i=2}^{n-2}\left(\begin{array}{c}
n \\
i
\end{array}\right) \frac{B_{i} B_{n-i}}{n-i}+\frac{B_{n}}{n}\right)=2 H_{n-1} \frac{B_{n}}{n} .
$$

Since $\frac{1}{n}\left(\frac{1}{i}+\frac{1}{n-i}\right)=\frac{1}{i(n-i)}$ and $\frac{2}{n^{2}}+\frac{2}{n} H_{n-1}=\frac{2}{n} H_{n}$, we can deduce (ii).

We note that some different proofs of Corollary 4 are already known using tools from combinatorics, $p$-adic analysis, contour integrals and other areas (see $[6,8,11,14,18]$ ).

Let $B_{n}^{\prime}:=\left(1-2^{n-1}\right) B_{n}$ for $n \geqslant 0$. Thus we see $B_{2 k+1}=0$ for all $k \geqslant 0$ and $B_{0}^{\prime}=1 / 2, B_{2}^{\prime}=-1 / 6, B_{4}^{\prime}=7 / 30, B_{6}^{\prime}=-31 / 42, B_{8}^{\prime}=127 / 30$ and so on.

Lemma 5. It follows that

$$
\begin{aligned}
& B_{0}(1 / 2)=1, \quad B_{n}(1 / 2)=\frac{1-2^{n-1}}{2^{n-1}} B_{n}=\frac{1}{2^{n-1}} B_{n}^{\prime} \quad(n \geqslant 1), \\
& G_{0}(1 / 2)=0, \quad G_{n}(1 / 2)=\frac{n}{2^{n-1}} E_{n-1} \quad(n \geqslant 1) .
\end{aligned}
$$

Proof. This lemma can be easily shown from the facts $P(2 t, 1 / 2)=2 P(t)-P(2 t)$ and $Q(2 t, 1 / 2)=2 t R(t)$.

Corollary 6. For $n \geqslant 4$, we have

$$
\begin{aligned}
& \sum_{i=2}^{n-2} B_{i}^{\prime} B_{n-i}^{\prime}-\frac{1}{n+2} \sum_{i=2}^{n-2}\left(\begin{array}{c}
n+2 \\
i
\end{array}\right) 2^{n-i} B_{i}^{\prime} B_{n-i}=\frac{n-1}{2} B_{n}^{\prime}+\frac{2^{n-1}}{n+2} B_{n}, \\
& \sum_{i=2}^{n-2} \frac{B_{i}^{\prime} B_{n-i}^{\prime}}{i(n-i)}-\frac{1}{n} \sum_{i=2}^{n-2}\left(\begin{array}{c}
n \\
i
\end{array}\right) \frac{2^{n-i} B_{i}^{\prime} B_{n-i}}{n-i}=H_{n} \frac{B_{n}^{\prime}}{n}+\frac{2^{n}-1}{n^{2}} B_{n} .
\end{aligned}
$$

Proof. We may assume that $n \geqslant 4$ is even by the same reason as mentioned in the proof of Corollary 4. Taking $x=1 / 2$ in Theorem 3-(i), we obtain from Lemma 5, noticing that $B_{n-1}=B_{n-1}^{\prime}=0$,

$$
\frac{1}{2^{n-2}} \sum_{i=2}^{n-2} B_{i}^{\prime} B_{n-i}^{\prime}-\frac{2}{n+2}\left(\sum_{i=2}^{n-2}\left(\begin{array}{c}
n+2 \\
i
\end{array}\right) \frac{B_{i}^{\prime} B_{n-i}}{2^{i-1}}+B_{n}\right)=\frac{n-1}{2^{n-1}} B_{n}^{\prime},
$$

which yields (i) multiplying by $2^{n-2}$. Similarly, taking $x=1 / 2$ in Theorem 3-(ii),

$$
\frac{1}{2^{n-2}} \sum_{i=}^{n-2} \frac{B_{i}^{\prime} B_{n-i}^{\prime}}{i(n-i)}-\frac{2}{n}\left(\sum_{i=2}^{n-2}\left(\begin{array}{c}
n \\
i
\end{array}\right) \frac{B_{i}^{\prime} B_{n-i}}{2^{i-1}(n-i)}+\frac{B_{n}}{n}\right)=H_{n-1} \frac{B_{n}^{\prime}}{2^{n-2} n} .
$$

Multiplying this by $2^{n-2}$ and replacing $H_{n-1}$ by $H_{n}-\frac{1}{n}$, we get (ii) as desired. 
We note that Corollary 6-(ii) is equivalent to the following identity discovered by Faber and Pandharipande in [9] with a proof by Zagier in an appendix:

$$
\sum_{i=1}^{n-1} \frac{\bar{B}_{i} \bar{B}_{n-i}}{i(n-i)}-\frac{1}{n} \sum_{i=1}^{n}\left(\begin{array}{l}
n \\
i
\end{array}\right) \frac{B_{i} \bar{B}_{n-i}}{i}=H_{n-1} \frac{\bar{B}_{n}}{n},
$$

where $\bar{B}_{i}:=\left(\left(1-2^{i-1}\right) / 2^{i-1}\right) B_{i}(i \geqslant 0)$. Indeed, we easily know that $\bar{B}_{i}=B_{i}^{\prime} / 2^{i-1}$ and $\bar{B}_{1}=\bar{B}_{n-1}=0$ for an even $n \geqslant 4$. Concerning this and other Miki type convolution identities, see also Dunne and Schubert [8], in which they presented a new and interesting approach using some tools from perturbative quantum field theory and string theory.

Similarly to Theorem 3, we can deduce the following identities using identity (3.2):

Theorem 7. For $n \geqslant 2$, we have

$$
\begin{aligned}
& \sum_{i=1}^{n-1} G_{i}(x) G_{n-i}(x)+\frac{4}{n+2} \sum_{i=0}^{n-2}\left(\begin{array}{c}
n+2 \\
i
\end{array}\right) B_{i}(x) G_{n-i}=0 \\
& \sum_{i=1}^{n-1} \frac{G_{i}(x) G_{n-i}(x)}{i(n-i)}+\frac{4}{n} \sum_{i=0}^{n-2}\left(\begin{array}{c}
n \\
i
\end{array}\right) \frac{B_{i}(x) G_{n-i}}{n-i}=0 .
\end{aligned}
$$

Proof. Since $G_{0}(x)=G_{0}=0$, identity (3.2) can be written as

$$
\begin{gathered}
\sum_{i=1}^{n-1}\left(\begin{array}{c}
n \\
i
\end{array}\right) u^{i}(1-u)^{n-i} G_{i}(x) G_{n-i}(x)=4 u(1-u) n B_{n-1}(x) \\
-2 \sum_{i=0}^{n-1}\left(\begin{array}{c}
n \\
i
\end{array}\right)\left(u^{n-i}(1-u)+u(1-u)^{n-i}\right) B_{i}(x) G_{n-i} .
\end{gathered}
$$

Integrating (3.5) between 0 and 1 with respect to $u$, we obtain using Lemma 2-(i), since $G_{1}=1$ and $\left(\frac{1}{n+1-i}-\frac{1}{n+2-i}\right)\left(\begin{array}{c}n \\ i\end{array}\right)=\frac{1}{(n+1-i)(n+2-i)}\left(\begin{array}{c}n \\ i\end{array}\right)=\frac{1}{(n+1)(n+2)}\left(\begin{array}{c}n+2 \\ i\end{array}\right)$,

$$
\begin{aligned}
\frac{1}{n+1} \sum_{i=1}^{n-1} G_{i}(x) G_{n-i}(x) & =\frac{2 n}{3} B_{n-1}(x)-4 \sum_{i=0}^{n-1}\left(\begin{array}{c}
n \\
i
\end{array}\right) \frac{B_{i}(x) G_{n-i}}{(n+1-i)(n+2-i)} \\
& =-\frac{4}{(n+1)(n+2)} \sum_{i=0}^{n-2}\left(\begin{array}{c}
n+2 \\
i
\end{array}\right) B_{i}(x) G_{n-i},
\end{aligned}
$$

which yields (i) multiplying by $n+1$. For identity (ii), dividing (3.5) by $u(1-u)$, we have

$$
\begin{aligned}
& \sum_{i=1}^{n-1}\left(\begin{array}{l}
n \\
i
\end{array}\right) u^{i-1}(1-u)^{n-1-i} G_{i}(x) G_{n-i}(x) \\
& \quad=4 n B_{n-1}(x)-2 \sum_{i=0}^{n-1}\left(\begin{array}{c}
n \\
i
\end{array}\right)\left(u^{n-1-i}+(1-u)^{n-1-i}\right) B_{i}(x) G_{n-i} \\
& \quad=-2 \sum_{i=0}^{n-2}\left(\begin{array}{c}
n \\
i
\end{array}\right)\left(u^{n-1-i}+(1-u)^{n-1-i}\right) B_{i}(x) G_{n-i} .
\end{aligned}
$$


Similarly to the above, integrating this between 0 and 1 with respect to $u$, we obtain using Lemma 2-(i),

$$
n \sum_{i=1}^{n-1} \frac{G_{i}(x) G_{n-i}(x)}{i(n-i)}=-4 \sum_{i=0}^{n-2}\left(\begin{array}{c}
n \\
i
\end{array}\right) \frac{B_{i}(x) G_{n-i}}{n-i},
$$

and so (ii) follows dividing by $n$.

As a consequence of Theorem 7, we will now derive two corollaries related to convolution identities for Genocchi and Euler numbers.

Corollary 8. For $n \geqslant 4$, we have

$$
\begin{aligned}
& \sum_{i=2}^{n-2} G_{i} G_{n-i}+4 \sum_{i=2}^{n-2}\left(\begin{array}{c}
n+1 \\
i-1
\end{array}\right) \frac{B_{i} G_{n-i}}{i}=-\frac{4}{n+2} G_{n}, \\
& \sum_{i=2}^{n-2} \frac{G_{i} G_{n-i}}{i(n-i)}+4 \sum_{i=2}^{n-2}\left(\begin{array}{c}
n-1 \\
i-1
\end{array}\right) \frac{B_{i} G_{n-i}}{i(n-i)}=-\frac{4}{n^{2}} G_{n} .
\end{aligned}
$$

Proof. Set $x=0$ in Theorem 7-(i). Noting that $G_{1}=B_{0}=1$ and $B_{1}=-1 / 2$, we have

$$
\begin{aligned}
& \sum_{i=1}^{n-1} G_{i} G_{n-i}+\frac{4}{n+2} \sum_{i=0}^{n-2}\left(\begin{array}{c}
n+2 \\
i
\end{array}\right) B_{i} G_{n-i} \\
& \quad=\sum_{i=2}^{n-2} G_{i} G_{n-i}+2 G_{n-1}+\frac{4}{n+2} \sum_{i=2}^{n-2}\left(\begin{array}{c}
n+2 \\
i
\end{array}\right) B_{i}(x) G_{n-i}+\frac{4}{n+2} G_{n}+4 B_{1} G_{n-1} \\
& \quad=\sum_{i=2}^{n-2} G_{i} G_{n-i}+4 \sum_{i=2}^{n-2}\left(\begin{array}{c}
n+2 \\
i-1
\end{array}\right) \frac{B_{i}(x) G_{n-i}}{i}+\frac{4}{n+2} G_{n}=0,
\end{aligned}
$$

and therefore (i) was shown. Similarly, to get identity (ii) we set $x=0$ in Theorem 7-(ii). Then, since $G_{1}=B_{0}=1$ and $B_{1}=-1 / 2$, we get

$$
\begin{aligned}
& \sum_{i=1}^{n-1} \frac{G_{i} G_{n-i}}{i(n-i)}+\frac{4}{n} \sum_{i=0}^{n-2}\left(\begin{array}{c}
n \\
i
\end{array}\right) \frac{B_{i} G_{n-i}}{n-i} \\
& =\sum_{i=2}^{n-2} \frac{G_{i} G_{n-i}}{i(n-i)}+\frac{2 G_{n-1}}{n-1}+\frac{4}{n} \sum_{i=2}^{n-2}\left(\begin{array}{c}
n \\
i
\end{array}\right) \frac{B_{i} G_{n-i}}{n-i}+\frac{4 G_{n}}{n^{2}}+\frac{4 B_{1} G_{n-1}}{n-1} \\
& =\sum_{i=2}^{n-2} \frac{G_{i} G_{n-i}}{i(n-i)}+4 \sum_{i=2}^{n-2}\left(\begin{array}{c}
n-1 \\
i-1
\end{array}\right) \frac{B_{i} G_{n-i}}{i(n-i)}+\frac{4 G_{n}}{n^{2}}=0,
\end{aligned}
$$

which gives (ii) as desired. 
Corollary 9. For $n \geqslant 4$, we have

$$
\begin{aligned}
& \sum_{i=1}^{n-1} i(n-i) E_{i-1} E_{n-1-i}+\sum_{i=2}^{n-2}\left(\begin{array}{c}
n+1 \\
i-1
\end{array}\right) 2^{n+1-i} \frac{B_{i}^{\prime} G_{n-i}}{i}=-\frac{2^{n}}{n+2} G_{n} \\
& \sum_{i=1}^{n-1} E_{i-1} E_{n-1-i}+\sum_{i=2}^{n-2}\left(\begin{array}{c}
n-1 \\
i-1
\end{array}\right) 2^{n+1-i} \frac{B_{i}^{\prime} G_{n-i}}{i(n-i)}=-\frac{2^{n}}{n^{2}} G_{n} .
\end{aligned}
$$

Proof. The above identities clearly follow if $n \geqslant 4$ is odd, and so we assume that $n \geqslant 4$ is even. Taking $x=1 / 2$ in Theorem 7 -(i), we obtain from Lemma 5 that, since $B_{0}(1 / 2)=1$ and $G_{n-1}=0$,

$$
\begin{aligned}
& \frac{1}{2^{n-2}} \sum_{i=1}^{n-1} i(n-i) E_{i-1} E_{n-1-i}+\frac{4}{n+2}\left(\sum_{i=2}^{n-2}\left(\begin{array}{c}
n+2 \\
i
\end{array}\right) \frac{1}{2^{i-1}} B_{i}^{\prime} G_{n-i}+G_{n}\right) \\
& =\frac{1}{2^{n-2}} \sum_{i=1}^{n-1} i(n-i) E_{i-1} E_{n-1-i}+4 \sum_{i=2}^{n-2}\left(\begin{array}{c}
n+1 \\
i-1
\end{array}\right) \frac{B_{i}^{\prime} G_{n-i}}{2^{i-1} i}+\frac{4}{n+2} G_{n}=0
\end{aligned}
$$

which yields (i) multiplying by $2^{n-2}$. Similarly to the above, taking $x=1 / 2$ in Theorem 7 (ii), we get, since $B_{0}(1 / 2)=1$ and $G_{n-1}=0$,

$$
\begin{aligned}
& \frac{1}{2^{n-2}} \sum_{i=1}^{n-1} E_{i-1} E_{n-1-i}+\frac{4}{n}\left(\sum_{i=2}^{n-2}\left(\begin{array}{c}
n \\
i
\end{array}\right) \frac{1}{2^{i-1}} \cdot \frac{B_{i}^{\prime} G_{n-i}}{n-i}+\frac{G_{n}}{n}\right) \\
& =\frac{1}{2^{n-2}} \sum_{i=1}^{n-1} E_{i-1} E_{n-1-i}+4 \sum_{i=2}^{n-2}\left(\begin{array}{c}
n-1 \\
i-1
\end{array}\right) \frac{1}{2^{i-1}} \cdot \frac{B_{i}^{\prime} G_{n-i}}{i(n-i)}+\frac{4 G_{n}}{n^{2}}=0 .
\end{aligned}
$$

Multiplying this identity by $2^{n-2}$, we can deduce (ii).

Applying identity (3.3), we can prove the following theorem:

Theorem 10. For $n \geqslant 2$, we have

$$
\begin{aligned}
& \sum_{i=1}^{n-1} B_{i}(x) G_{n-i}(x)-\sum_{i=1}^{n-2}\left(\begin{array}{c}
n+1 \\
i-1
\end{array}\right) \frac{2^{n-i} G_{i}(x) B_{n-i}}{i}=\frac{n-1}{2} G_{n}(x) \\
& \sum_{i=1}^{n-1} \frac{B_{i}(x) G_{n-i}(x)}{i(n-i)}-\sum_{i=1}^{n-2}\left(\begin{array}{c}
n-1 \\
i-1
\end{array}\right) \frac{2^{n-i} G_{i}(x) B_{n-i}}{i(n-i)}=H_{n-1} \frac{G_{n}(x)}{n} .
\end{aligned}
$$

Proof. Since $G_{0}=G_{0}(x)=0$, identity (3.3) can be written as

$$
\begin{aligned}
& \sum_{i=0}^{n-1}\left(\begin{array}{c}
n \\
i
\end{array}\right) u^{i}(1-u)^{n-i} B_{i}(x) G_{n-i}(x)=u(1-u) n G_{n-1}(x) \\
& \quad+(1-u) \sum_{i=1}^{n}\left(\begin{array}{c}
n \\
i
\end{array}\right) u^{n-i} G_{i}(x) B_{n-i}-\frac{u}{2} \sum_{i=1}^{n-1}\left(\begin{array}{c}
n \\
i
\end{array}\right)(1-u)^{n-i} G_{i}(x) G_{n-i} .
\end{aligned}
$$


Gathering the terms involving $G_{n}(x)$ in one place, we have, since $B_{0}=B_{0}(x)=1$,

$$
\begin{aligned}
& \sum_{i=1}^{n-1}\left(\begin{array}{c}
n \\
i
\end{array}\right) u^{i}(1-u)^{n-i} B_{i}(x) G_{n-i}(x) \\
& =u(1-u) n G_{n-1}(x)+\left((1-u)-(1-u)^{n}\right) G_{n}(x) \\
& \quad+(1-u) \sum_{i=1}^{n-1}\left(\begin{array}{c}
n \\
i
\end{array}\right) u^{n-i} G_{i}(x) B_{n-i}-\frac{u}{2} \sum_{i=1}^{n-1}\left(\begin{array}{c}
n \\
i
\end{array}\right)(1-u)^{n-i} G_{i}(x) G_{n-i}
\end{aligned}
$$

Integrating (3.6) between 0 and 1 with respect to $u$, we obtain using Lemma 2, since $G_{n-i}=2\left(1-2^{n-i}\right) B_{n-i}$ and $B_{1}-\frac{1}{2} G_{1}=-1$,

$$
\begin{aligned}
& \frac{1}{n+1} \sum_{i=1}^{n-1} B_{i}(x) G_{n-i}(x)=\frac{n}{6} G_{n-1}(x)+\frac{n-1}{2(n+1)} G_{n}(x) \\
& \quad+\sum_{i=1}^{n-2}\left(\begin{array}{c}
n \\
i
\end{array}\right) \frac{G_{i}(x)\left(B_{n-i}-\frac{1}{2} G_{n-i}\right)}{(n+1-i)(n+2-i)}+\left(\begin{array}{c}
n \\
n-1
\end{array}\right) \frac{G_{n-1}(x)\left(B_{1}-\frac{1}{2} G_{1}\right)}{2 \cdot 3} \\
& =\frac{n-1}{2(n+1)} G_{n}(x)+\frac{1}{n+1} \sum_{i=1}^{n-2}\left(\begin{array}{c}
n+1 \\
i-1
\end{array}\right) \frac{2^{n-i} G_{i}(x) B_{n-i}}{i},
\end{aligned}
$$

which leads to (i) multiplying by $n+1$. Next, dividing (3.6) by $u(1-u)$, we have

$$
\begin{aligned}
& \sum_{i=1}^{n-1}\left(\begin{array}{c}
n \\
i
\end{array}\right) u^{i-1}(1-u)^{n-1-i} B_{i}(x) G_{n-i}(x)=n G_{n-1}(x)+\frac{1-(1-u)^{n-1}}{u} G_{n}(x) \\
& \quad+\sum_{i=1}^{n-1}\left(\begin{array}{c}
n \\
i
\end{array}\right) u^{n-1-i} G_{i}(x) B_{n-i}-\frac{1}{2} \sum_{i=1}^{n-1}\left(\begin{array}{c}
n \\
i
\end{array}\right)(1-u)^{n-1-i} G_{i}(x) G_{n-i} .
\end{aligned}
$$

Similarly to the above, integrating this from 0 to 1 with respect to $u$, we obtain using again Lemma 2,

$$
\begin{aligned}
& n \sum_{i=1}^{n-1} \frac{B_{i}(x) G_{n-i}(x)}{i(n-i)}=n G_{n-1}(x)+H_{n-1} G_{n}(x) \\
& \quad+\sum_{i=1}^{n-2}\left(\begin{array}{c}
n \\
i
\end{array}\right) \frac{G_{i}(x)\left(B_{n-i}-\frac{1}{2} G_{n-i}\right)}{n-i}+n G_{n-1}(x)\left(B_{1}-\frac{1}{2} G_{1}\right) \\
& =H_{n-1} G_{n}(x)+\sum_{i=1}^{n-2}\left(\begin{array}{c}
n \\
i
\end{array}\right) 2^{n-i} \frac{G_{i}(x) B_{n-i}}{n-i}
\end{aligned}
$$

which gives (ii) dividing by $n$. 
Corollary 11. For $n \geqslant 4$, we have

$$
\begin{aligned}
& \sum_{i=2}^{n-2} B_{i} G_{n-i}-\sum_{i=2}^{n-2}\left(\begin{array}{c}
n+1 \\
i-1
\end{array}\right) \frac{2^{n-i} G_{i} B_{n-i}}{i}=\frac{n-1}{2} G_{n} \\
& \sum_{i=2}^{n-2} \frac{B_{i} G_{n-i}}{i(n-i)}-\sum_{i=2}^{n-2}\left(\begin{array}{c}
n-1 \\
i-1
\end{array}\right) \frac{2^{n-i} G_{i} B_{n-i}}{i(n-i)}=H_{n-1} \frac{G_{n}}{n}
\end{aligned}
$$

Proof. Both identities immediately follow from Theorem 10 putting $x=0$, because $B_{n-1}=G_{n-1}=0$ for an even $n \geqslant 4$.

Corollary 12. For $n \geqslant 3$, we have

$$
\begin{aligned}
& \sum_{i=2}^{n-1}(n-i) B_{i}^{\prime} E_{n-1-i}-\sum_{i=1}^{n-2}\left(\begin{array}{c}
n+1 \\
i-1
\end{array}\right) 2^{2(n-i)-1} E_{i-1} B_{n-i}=\frac{n(n-1)}{4} E_{n-1}, \\
& \sum_{i=2}^{n-1} \frac{B_{i}^{\prime} E_{n-1-i}}{i}-\sum_{i=1}^{n-2}\left(\begin{array}{c}
n-1 \\
i-1
\end{array}\right) \frac{2^{2(n-i)-1} E_{i-1} B_{n-i}}{n-i}=\frac{1}{2} H_{n-1} E_{n-1} .
\end{aligned}
$$

Proof. If $n \geqslant 3$ is even, then the above identities are trivial because both sides of (i) and (ii) entirely vanish. Hence we assume that $n \geqslant 3$ is odd. Taking $x=1 / 2$ in Theorem 10(i), we obtain from Lemma 5 that

$$
\frac{1}{2^{n-2}} \sum_{i=1}^{n-1}(n-i) B_{i}^{\prime} E_{n-1-i}-\sum_{i=1}^{n-2}\left(\begin{array}{c}
n+1 \\
i-1
\end{array}\right) 2^{n+1-2 i} E_{i-1} B_{n-i}=\frac{n(n-1)}{2^{n}} E_{n-1} .
$$

Multiplying this by $2^{n-2}$ and noting $B_{1}^{\prime}=0$, we can deduce (i). In the same way as above, taking $x=1 / 2$ in Theorem 10 -(ii), we have

$$
\frac{1}{2^{n-2}} \sum_{i=1}^{n-1} \frac{B_{i}^{\prime} E_{n-1-i}}{i}-\sum_{i=1}^{n-2}\left(\begin{array}{c}
n-1 \\
i-1
\end{array}\right) \frac{2^{n+1-2 i} E_{i-1} B_{n-i}}{n-i}=\frac{1}{2^{n-1}} H_{n-1} E_{n-1},
$$

which gives (ii) multiplying by $2^{n-2}$.

\section{Additional remarks}

In the above arguments, we did not ostensibly treat the M-M-G type convolution identities for Euler polynomials. To discuss and deduce them, we may apply the following functional equation satisfied by the generating function $R(t, x)$ of $E_{n}(x)$ given in (1.3): letting $u, v \in$ $\mathbb{R} \backslash\{0\}$ with $u+v \neq 0$ and $W$ be as in Theorem 1 ,

$$
\begin{aligned}
t R(u t, x) R(v t, y)= & \frac{4}{u+v} P((u+v) t, W)-\frac{2}{u+v} P((u+v) t, W) R(u t) \\
& -\frac{2}{u+v} P((u+v) t, W) R(v t) .
\end{aligned}
$$


However, since $Q(t, x)=t R(t, x)$, we know $G_{n}(x)=n E_{n-1}(x)(n \geqslant 1)$ and this fact tells that Genocchi polynomials can be expressed in terms of Euler polynomials and vice versa. In conclusion, it suffices to deal with either Genocchi or Euler polynomials, and therefore, we chose and discussed the former polynomials in this paper.

\section{Acknowledgements}

The author wishes to thank the anonymous referee for his/her valuable comments and suggestions which resulted in an improved presentation of the paper.

\section{References}

[1] T. Agoh, On Bernoulli numbers, I, C. R. Math. Acad. Sci. Soc. R. Can., 10 (1988), $7-12$.

[2] T. Agoh and K. Dilcher, Convolution identities and lacunary recurrences for Bernoulli numbers, J. Number Theory, 124 (2007), 105-122.

[3] T. Agoh and K. Dilcher, Higher-order recurrences for Bernoulli numbers, J. Number Theory, 129 (2009), 1837-1847.

[4] K. W. Chen, Sums of products of generalized Bernoulli polynomials, Pacific J. Math., 208 (2003), 39-52.

[5] W. Chu and R. Zhou, Convolutions of Bernoulli and Euler polynomials, Saraevo J. Math., 6 (2010), 147-163.

[6] M. C. Crabb, The Miki-Gessel Bernoulli number identity, Glasg. Math. J., 47 (2005), $327-328$.

[7] K. Dilcher, Sums of products of Bernoulli numbers, J. Number Theory, 60 (1996), 23-41.

[8] G. V. Dunne and C. Schubert, Bernoulli number identities from quantum field theory, Preprint, 2014. arXiv:math.nt/0406610

[9] C. Faber and R. Pandharipande, Hodge integrals and Gromov-Witten theory, Invent. Math., 139 (2000), 173-199.

[10] A. M. Fu, H. Pan and F. Zhang, Symmetric identities on Bernoulli polynomials, Preprint, 2007. arXiv:0709.2593

[11] H. G. Gadiyar and R. Padma, A comment on Matiyasevich's identity \#0102 with Bernoulli numbers, Preprint, 2006. arXiv:math/0608675

[12] I. M. Gessel, On Miki's identity for Bernoulli numbers, J. Number Theory, 110 (2005), $75-82$.

[13] E. R. Hansen, "A Table of series and products", Prentice-Hall, Inc., Englewood Cliffs, NJ, 1975.

[14] S. Hu, D. Kim and M-S. Kim, New identities involving Bernoulli, Euler and Genocchi numbers, Adv. Difference Equ., 2013, 2013:74. 
[15] Y. Matiyasevich, Identities with Bernoulli numbers, Idnetity \#0202, 1997. http://logic.pdmi.ras.ru/ yumat/Journal/Bernoulli/bernulli.htm

[16] H. Miki, A relation between Bernoulli numbers, J. Number Theory, 10 (1978), 297302.

[17] H. Pan and Z.W. Sun, New identities involving Bernoulli and Euler polynomials, $J$. Combin. Theory Ser. A, 113 (2006), 156-175.

[18] K. Shiratani and S. Yokoyama, An application of p-adic convolutions, Mem. Fac. Sci. Kyushu Univ. Ser. A, 36 (1982), 73-83. 